\title{
PROSPECTIVE WORK FOR ALMA, HERSCHEL, SOFIA: SUBMILLIMETER-WAVE SPECTROSCOPY OF COMPLEX ORGANIC MOLECULES
}

\author{
T.R. Huet ${ }^{1}$, L. Margulès ${ }^{1}$, R.A. Motiyenko ${ }^{1}$, S. Bailleux ${ }^{1}$, M. Goubet $^{1}$ \\ and G. Wlodarczak ${ }^{1}$
}

\begin{abstract}
Laboratory analysis of spectra of potential interstellar molecules from the radio-frequency to the $\mathrm{THz}$ range is a prerequisite for their subsequent identification by radioastronomical techniques. The spectral analysis allows us to derive molecular parameters that are able to model the frequency and intensity patterns of the transitions, as well as to predict with a high degree of precision the frequencies of transitions not measured in the laboratory.
\end{abstract}

\section{Introduction}

This paper summarizes the recent results obtained in Lille on the laboratory spectroscopy of the most abundant isotopologues species of methyl formate and glycolaldehyde, two isomers of $\mathrm{C}_{2} \mathrm{H}_{4} \mathrm{O}_{2}$. We mainly focus on the experimental devices. The astrophysical study of molecular isotopologues of complex organic molecules is important for several reasons. First it gives access to the isotopic abundances in astronomical environments. Second it allows the astronomers to discriminate in their surveys the transition lines due to the isotopologues and eventually eliminate them to be able to discover new species. Indeed the upcoming ALMA and the present Herschel sub-mm facilities will provide large amounts of high precision $(\Delta \nu \leq 1 \mathrm{MHz})$ spectroscopic data in the wavelength range down to $150 \mu \mathrm{m}$ (or up to $2 \mathrm{THz}$ ). The interferometer ALMA, in addition, will provide a so far unprecedented spatial resolution and a sensitivity which reaches the limit of line confusion. Both HIFI/Herschel and ALMA provide a wealth of data on dense clouds with young stars and surrounding material that contain molecular species with numerous and strong transitions throughout the whole sub-mm region. Much of the line

1 Laboratoire PhLAM, Bât. P5, CNRS UMR 8523, Université Lille 1, 59655 Villeneuve d'Ascq Cedex, France; e-mail: therese.huet@univ-lille1.fr 
emission comes from known species, such as methyl formate and similarly complex molecules. Without spectral identifications, however, the resulting forest of spectral lines will seriously hinder the detection and analysis of new molecular and radical species. This problem is known as the "Weeds and Flowers problem". For an optimal science exploitation of ALMA and Herschel it is necessary to cut down the weeds and to recognize the flowers. Methyl formate and its isotopologues are classified as "weed, class I" species due to their abundance in observations. Third, the observation of isotopologue transitions is needed to derive correct column densities for abundant molecules exhibiting optically thick lines, which could be the case for methyl formate in some hot cores.

\section{Experiment}

\subsection{Microwave spectroscopy}

A new molecular beam Fourier Transform Microwave spectrometer has been developed in Lille (Tudorie et al. 2011). The main improvement of the present experimental setup over the old one (Kassi et al. 2000) is an increase of the mirrors diameter from 0.4 to $0.7 \mathrm{~m}$ leading to an improved signal-to-noise ratio at low frequencies and to less diffraction losses. Signals can be recorded in the $2-20 \mathrm{GHz}$ spectral region. The sample vapors at a pressure of about 10 mbar are mixed with neon carrier gas at a backing pressure of $1-2$ bar. The mixture is introduced into a Fabry-Perot cavity at a repetition rate of $1.5 \mathrm{~Hz}$. Molecules are polarized within the supersonic expansion by a $2 \mu$ s pulse generated by an Agilent E8257D synthesizer and the free induction decay signal is detected and digitized at a repetition rate of $120 \mathrm{MHz}$. After transformation of the time domain signal, molecular lines are observed as Doppler doublets, with a signal point every $0.46 \mathrm{kHz}$ corresponding to a data accumulation of $2^{18}$ points. Each average spectrum is obtained by coadding about $100-1000$ signals. Transition frequencies are measured as the mean value of the two Doppler components and for most lines the uncertainty of the measurements is estimated to be less than $1 \mathrm{kHz}$. The Doppler width is a few $\mathrm{kHz}$.

\subsection{Millimeter-wave and submillimeter-wave spectroscopy}

The millimeter- and submillimeter-wave measurements $(75-990 \mathrm{GHz})$ are mainly performed using the Lille spectrometer based on solid-state sources (Motiyenko et al. 2010). The frequency of an Agilent synthesizer E8257D $(12.5-18.4 \mathrm{GHz})$ is first multiplied by six and amplified by a Spacek active sextupler, providing an output power of $+15 \mathrm{dBm}$ in the $\mathrm{W}$-band range $(75-110 \mathrm{GHz})$. This power is high enough to use passive Schottky multipliers $(\times 2, \times 3, \times 5, \times 3 \times 2, \times 3 \times 3)$ from Virginia Diodes Inc. in the next stage of the frequency multiplication chain. As a detector we use an InSb liquid He-cooled bolometer from QMC Instruments Ltd. To improve the sensitivity of the spectrometer, the sources are frequency modulated at $10 \mathrm{kHz}$ and phase-sensitive detection at $2 \mathrm{f}$ is employed. The absorption 
cell is a stainless-steel tube (6 $\mathrm{cm}$ diameter, $220 \mathrm{~cm} \mathrm{long})$. The sample pressure during measurements is about $2 \mathrm{~Pa}$ and the linewidth is limited by Doppler broadening. Measurements are performed at room temperature. The accuracy of the measurements for isolated lines is estimated to be better than $30 \mathrm{kHz}$. However, if the lines are blended or have a poor signal-to-noise ratio, they are given a weight of $100 \mathrm{kHz}$.

\section{Theoretical models}

Like many others complex organic molecules, methyl formate exhibits large amplitude motion, i.e. the internal rotation of the methyl group with respect to the molecule. As the molecule is light and as the barrier to internal rotation is moderate $\left(\mathrm{V}_{3} \approx 389 \mathrm{~cm}^{-1}\right.$ for the normal species), the internal rotation splittings are relatively large, and reach values up to a few $\mathrm{MHz}$ in the torsional ground state. Therefore an appropriate theory is needed to model and calculate with accuracy the transition frequencies, especially at high $\mathrm{J}$ values. Also the assigments and analysis of the spectra are not obvious.

The description of the theories used for modeling our spectra can be found in (Carvajal et al. 2009) and (Margulès et al. 2009) respectively for the symmetric $\left(\mathrm{CH}_{3}\right)$ and asymmetric $\left(\mathrm{CH}_{2} \mathrm{D}\right.$ or $\left.\mathrm{CHD}_{2}\right)$ methyl rotor.

In the case of the symmetric methyl rotor, each rotational line is split into a doublet (characterized by the symmetry labels $\mathrm{A}$ and $\mathrm{E}$, the $\mathrm{E}$ line being doubly degenerate). In the case of the asymmetric methyl rotor, each rotational line is split into three lines: one is due to the in-plane conformer, and the two others are due to the internal rotation splitting for the two equivalent out-of-plane conformers.

Glycolaldehyde is an asymmetric top containing an hydroxyl (OH) group, which is stabilized by an intramolecular hydrogen bond. Therefore any spectrum can modelled with a set of molecular parameters containg the principal rotation constants, and centrifugal distortion parameters, up to the octic terms.

\section{Results}

\subsection{Laboratory spectroscopy}

Concerning methyl formate, several isotopologues have been characterized in our Laboratory. The $\mathrm{H}^{13} \mathrm{COOCH}_{3}, \mathrm{HCOO}^{13} \mathrm{CH}_{3}$ (Carvajal et al. 2009), $\mathrm{DCOOCH}_{3}$ (Margulès et al. 2010), $\mathrm{HC}^{18} \mathrm{OOCH} 3, \mathrm{HCO}^{18} \mathrm{OCH} 3$ (Tercero et al. 2012), $\mathrm{HCOOCH}_{2} \mathrm{D}$ (Margulès et al. 2009), and $\mathrm{HCOOCHD}_{2}$ (Coudert et al. 2012) ground state spectra have been analyzed and modeled with high accuracy, in order to predict any transition frequency within $100 \mathrm{KHz}$.

Concerning glycolaldehyde, we have measured the spectra of deuterated isotopologues in the laboratory: the three mono-deuterated ones $\left(\mathrm{CH}_{2} \mathrm{OD}-\mathrm{CHO}\right.$, $\mathrm{CHDOH}-\mathrm{CHO}$ and $\mathrm{CH}_{2} \mathrm{OH}-\mathrm{CDO}$ ) and one dideuterated derivative (CHDOH-CDO) in the ground vibrational state (Bouchez et al. 2012). 


\subsection{Detection in Orion}

Several isotopologues species of methyl formate have been detected in the interstellar medium so far. Indeed the $\mathrm{H}^{13} \mathrm{COOCH}_{3}, \mathrm{HCOO}^{13} \mathrm{CH}_{3}$ (Carvajal et al. 2009), $\mathrm{DCOOCH}_{3}$ (Margulès et al. 2010), $\mathrm{HC}^{18} \mathrm{OOCH}_{3}$, and $\mathrm{HCO}^{18} \mathrm{OCH}_{3}$ (Tercero et al. 2012) species have been detected toward Orion KL (B. Tercero, N. Marcelino and J. Cernicharo, CSIC-INTA, Madrid, Spain).

\section{Conclusion}

The PhLAM Laboratory has developed over the years a unique set of instrumentation dedicated to the microwave, millimeter-wave and submillimeter-wave spectroscopy of astrophysical molecules. Our very high resolution spectrometers cover the $2-990 \mathrm{GHz}$ range. Future developments include a new multiplier chain based on solid state sources in order to cover the $1.1-1.7 \mathrm{THz}$ spectral range. The AILES beam line at the third generation synchrotron radiation SOLEIL was recently proven to be highly complementary to our spectrometers, as shown for the NH radical study by Bailleux et al. (2012).

The authors gratefully acknowledge J.-C. Guillemin (ENSCR), H. Møllendal (Oslo), L. Coudert and I. Kleiner (LISA) for fruitful collaboration in laboratory work. This work was supported by the French contracts ANR-08-BLAN-0054, ANR-08-BLAN-0225, the Action sur projet INSU Physique et Chimie du Milieu Interstellaire (PCMI-CNRS), and the Centre National d'Études Spatiales (CNES).

\section{References}

Bailleux, S., Martin-Drumel, M.A., Margulès L., et al., 2012, A\&A, 538, 135

Bouchez, A., Margulès, L., Motiyenko, R.A., et al., 2012, A\&A, 540, 51

Carvajal, M., Margulès, L., Tercero, B., et al., 2009, A\&A, 500, 1109

Coudert, L.H., Margulès, L., Huet, T.R., et al., 2012, A\&A, 543, 46

Kassi, S., Petitprez, D., \& Wlodarczak, G., 2000, J. Mol. Struct., 517, 375

Margulès, L., Coudert, L.H., Møllendal, H., et al., 2009, J. Mol. Spectrosc., 254, 55

Margulès, L., Huet, T.R., Demaison, J., et al., 2010, ApJ, 714, 1120

Motiyenko, R.A., Margulès, L., Alekseev, E.A., et al., 2010, J. Mol. Spectrosc., 264, 94

Tercero, B., Margulès, L., Coudert, L.H., Carvajal, M., et al., 2012, A\&A, 538, 119

Tudorie, M., Coudert, L.H., Huet, T.R., et al., 2011, J. Chem. Phys., 134, 074314 